\title{
NUSAPI Accelerates Osteosarcoma Cell Proliferation and Cell Cycle Progression via Upregulating CDC20 and Cyclin A2
}

\author{
Haoran Wang' \\ Zixiang Liu' \\ Peng $\mathrm{Wu}^{\mathrm{I}}$ \\ Hanqing Wang' \\ Weiwei Ren ${ }^{2}$ \\ 'Department of Orthopedics, Hangzhou \\ Children's Hospital, Hangzhou, People's \\ Republic of China; ${ }^{2}$ Department of \\ Gynecology, Hangzhou Children's \\ Hospital, Hangzhou, People's Republic of \\ China
}

\begin{abstract}
Purpose: Nucleolar and spindle-associated protein 1 (NUSAP1) is a significant mitotic regulator and has been found to be implicated in carcinogenesis of several cancers. The aim of this study was to explore the functional role and underlying mechanisms of NUSAP1 in osteosarcoma.
\end{abstract}

Methods: Western blot assay and Real-time fluorescent quantitative polymerase chain reaction (RT-qPCR) were employed to assess the expressions of NUSAP1, cell division cycle 20 homologue (CDC20) and cyclin A2 (CCNA2) in osteosarcoma cells. Cell proliferation was evaluated by Cell Counting Kit-8 (CCK-8) assay and 5-ethynyl-2'-deoxyuridine (EdU) assay, and flow cytometry was applied for exploring cell cycle. In addition, an osteosarcoma tumor-bearing mouse model was established by injection of transfected osteosarcoma cells. Tumor volume and protein expressions of Ki67 and PCNA were examined. Bioinformatics analysis and immunoprecipitation were used to identify the combination of NUSAP1 with CDC20 and CCNA2.

Results: The mRNA and protein expression of NUSAP1 were extremely upregulated in osteosarcoma cells. Overexpression of NUSAP1 promoted whereas NUSAP1 silencing suppressed cell proliferation and cell cycle progression in transfected osteosarcoma cells. In osteosarcoma mouse model, NUSAP1 expression affected tumor volume and levels of Ki67 and PCNA. Moreover, CDC20 or CCNA2 silencing inhibited NUSAP1-induced cell proliferation and cell cycle in osteosarcoma cells.

Conclusion: Our data demonstrated that upregulated NUSAP1 may exacerbate the development of osteosarcoma by accelerating the proliferation and cell cycle process of osteosarcoma cells by binding to CDC20 and CCNA2, suggesting NUSAP1 as a possible therapeutic target for treatment of osteosarcoma.

Keywords: NUSAP1, osteosarcoma, CDC20, cyclin A2, cell cycle
Correspondence: Weiwei Ren Department of Gynecology, Hangzhou Children's Hospital, No. 195 Wenhui

Road, Hangzhou, Zhejiang Province, People's Republic of China

Email rwwdoc086@I63.com

\section{Introduction}

Osteosarcoma is a primary malignant bone tumor characterized by osteoid tissue or the formation of immature bone in childhood and adolescence. ${ }^{1-3}$ In the past, all patients suffering from osteosarcoma need to be amputated forcedly and the cure rate is less than $10 \%$. Today, with the advantages of surgery technology and medical equipment, the percentage of patients cured is raised to $60 \%$. ${ }^{4}$ However, detectable metastases were found in $15 \%$ to $20 \%$ of patients, among which $85 \%$ of metastatic disease occurs in the lung. ${ }^{4,5}$ The overall 5-year survival rate has not been improved on account of metastasis and the tolerance to chemotherapies. ${ }^{6}$ 
Thus, it is of great clinical value to further understand molecular mechanisms of osteosarcoma and develop more effective therapeutic strategies for cure of osteosarcoma.

Nucleolar and spindle-associated protein 1 (NUSAP1), as an indispensable mitotic regulator, takes part in cellular process of mitosis including spindle assembly, cytokinesis, spindle formation and stability. ${ }^{7-9}$ Previous studies have reported that when the depletion of NUSAP1 that is expressed selectively in cellular proliferation process occurred, multiple abnormal cellular events were observed such as decreased microtubules numbers in central spindle, aberrant chromosomal segregation and spindle assembly, G2/M arrest, and chromosomal misalignment. ${ }^{10,11}$ These disordered movements of mitosis are important factors for occurrence of many cancers. ${ }^{12}$ NUSAP1 has been discovered to be associated with tumorigenesis in many types of cancers. ${ }^{8}$ Upregulation of NUSAP1 was observed in several cancers, such as breast cancer, ${ }^{13}$ prostate cancer ${ }^{10}$ and hepatocellular carcinomas, ${ }^{14}$ which provides evidence of involvement of NUSAP1 in cancer development and metastasis. However, little is known about the expression and roles of NUSAP1 in human osteosarcoma.

In the current study, we revealed the high expression of NUSAP1 in several osteosarcoma cell lines. Alteration of NUSAP1expression regulated cell proliferation and cell cycle progress in osteosarcoma cells by binding to cell division cycle 20 homologue (CDC20) and cyclin A2 (CCNA2), providing a potential novel mechanism for therapeutic strategy for osteosarcoma.

\section{Cell Culture}

Human normal osteoblast cell line hFOB1.19 and osteosarcoma cell lines MG63, U2OS and 143B were purchased from the CCTCC (China Center for Type Culture Collection, Wuhan, China). These cells were cultured in Dulbecco's modified Eagle's medium (DMEM) containing 10\% fetal bovine serum (FBS; Thermo Fisher Scientific), $100 \mu \mathrm{g} / \mathrm{mL}$ streptomycin and $100 \mathrm{U} / \mathrm{mL}$ penicillin with $5 \%$ $\mathrm{CO}_{2}$ incubator at $37^{\circ} \mathrm{C}$.

\section{Cell Transfection}

NUSAP1-specific pcDNA overexpression vector (OvNUSAP1), shRNA-NUSAP1-1/2, shRNA-CDC20-1/2, shRNA-CCNA2-1/2 and corresponding negative controls shRNA-NC, Ov-NC were purchased from Invitrogen (Nanjing, China). Osteosarcoma cells were transfected with the plasmid vectors by Lipofectamine 2000
(Thermo Fisher Scientific, Inc.) in accordance with manufacturer's guidance.

\section{Cell Counting Kit-8 (CCK-8) Assay}

Osteosarcoma cells transfected with shRNA-NUSAP1-1 or Ov-NUSAP1 and shRNA-CDC20/shRNA-CCNA2 were plated in 96-well plates, and were cultured for 24 h. Then, $10 \mu \mathrm{L}$ CCK-8 reagent (Dojindo, Japan) was added into the transfected cells. After $2 \mathrm{~h}$ incubation, the absorbance of each well at $450 \mathrm{~nm}$ was detected by microplate reader (Bio-Rad Laboratories, Inc., Hercules, CA, USA). Each group was replicated for five times and all assays were independently repeated three times.

\section{5-Ethynyl-2'-Deoxyuridine (EdU) Assay}

EdU assay was performed to assess cell proliferation of the osteosarcoma cells. The transfected cells were added with EdU $(50 \mu \mathrm{M})$ and incubated at $37^{\circ} \mathrm{C}$ for $4 \mathrm{~h}$. After fixation with $4 \%$ formaldehyde for $15 \mathrm{~min}$ and permeabilized with $0.5 \%$ Triton X-100 for $20 \mathrm{~min}$ at room temperature, the cells were incubated with $100 \mu \mathrm{L}$ of $1 \times$ Apollo $^{\circledR}$ reaction cocktail for $30 \mathrm{~min}$. Finally, cells were stained with100 $\mu \mathrm{L}$ of Hoechst 33,342 for $30 \mathrm{~min}$ and visualized with fluorescence microscopy (Bio-Rad, Japan).

\section{RT-qPCR}

Total RNA from osteosarcoma cells MG63, U2OS and 143B and osteoblast cell line hFOB1.19 were extracted by TRIzol Reagent (Invitrogen, Carlsbad). Following the detection of purity and concentration of RNA by NanoDrop 3000 (ThermoScientific, MA) at 260/280 nm, the RNA was converted to cDNA by PrimeScript RT Master Mix (Takara Bio, Inc., Tokyo, Japan). Amplification of the cDNA was carried out by real-time quantitative PCR using the SYBR Premix Ex Taq ${ }^{\text {TM }}$ II kit (Takara, Shiga, Japan) on an ABI PRISM 7900 Real-Time system (Applied Biosystems, Foster City, CA, USA). GAPDH was used as the internal control for NUSAP1, CDC20 and CCNA2.

\section{Western Blot Analysis}

RIPA buffer was utilized to extract proteins from transfected MG63, U2OS, 143B h and FOB1.19. The protein concentrations were then measured by BCA Protein Assay kit (Pierce Biotechnology). Total protein samples were separated by $10 \%$ SDS-PAGE and transferred onto PVDF membranes (Invitrogen). Membranes were incubated with the primary antibodies against NUSAP1 (1 
$\mu \mathrm{g} / \mathrm{mL}, \quad \mathrm{ab} 169083), \quad \operatorname{Ki67}$ (1:1000, ab16667), PCNA (1:1000, ab92552), CDC20 (1:2000, ab183479), CCNA2 (1:2000, ab181591) and GAPDH (1:2500, ab9485) overnight at $4{ }^{\circ} \mathrm{C}$ and incubated with HRP-conjugated secondary antibodies for $2 \mathrm{~h}$. Finally, the bands on the membrane were visualized with enhanced chemiluminescence (ECL) Western Blotting System (GE Healthcare) and quantified with Image $\mathrm{J}$ software.

\section{Xenograft Experiments}

All procedures using animals were approved by Animal Care and Use Committee of Hangzhou Children's Hospital. All animal studies were conducted with the guidelines established by the Animal Care and Use Committee of Hangzhou Children's Hospital. To establish an in vivo osteosarcoma model, $143 \mathrm{~B}$ cells $\left(5 \times 10^{6}\right.$ cells $)$ transfected with Ov-NUSAP1 or shRNA-NUSAP1-1 and control cells were subcutaneously injected into the nude mice on flanks. Tumor volume was measured every two days for two weeks. At the 14th day after infection, the mice were sacrificed with cervical dislocation. The tumors were harvested and stripped, and were homogenized for Western blot assay.

\section{Flow Cytometry}

Flow cytometric analysis was implemented to measure cell cycle distribution. Briefly, the adhered cells transfected different plasmids were obtained by trypsinization and fixed with $70 \%$ ethanol. Then, cells were stained with 50 $\mu \mathrm{g} / \mathrm{mL}$ propidium iodide and $100 \mu \mathrm{g} / \mathrm{mL}$ RNase I in PBS for $30 \mathrm{~min}$ at $37^{\circ} \mathrm{C}$. The samples were analyzed with a FACScan flow cytometer (BD Biosciences, San Jose, CA) and BD CellQuest software (BD Bioscience, USA).

\section{Immunoprecipitation (IP) Assay}

Osteosarcoma 143B cells were seeded in 12-well plates and harvested at $80-90 \%$ confluency. Then the cells were lysed in immunoprecipitation lysis buffer and were centrifuged at $15,000 \mathrm{~g}$ for $10 \mathrm{~min}$. The supernatants were incubated with antibodies against NUSAP1, CDC20 and CCNA2 at $4^{\circ} \mathrm{C}$. Protein A-Sepharose was used to precipitate with the immunocomplexes and the complexes were analyzed by SDS-PAGE using ECL system detection.

\section{Statistical Analysis}

SPSS 17.0 (IBM, Armonk, NY, USA) was executed to analyze data. Data are presented as the means \pm S.D. A paired or unpaired $t$-test was used to evaluate statistical significance of the differences in two groups, and the differences among over two groups were analyzed by one-way ANOVA. $\mathrm{P}<0.05$ represents a statistically significant difference.

\section{Results \\ NUSAPI Was Upregulated in Osteosarcoma Cell Lines}

To investigate the role of NUSAP1 in osteosarcoma, we examined the expression of NUSAP1 in several osteosarcoma cells and control cells. As shown in Figure 1A and B, the mRNA and protein level of NUSAP1 were significantly increased in MG63, U2OS and 143B cells compared with that in the normal osteoblast cell line hFOB1.19. Given that NUSAP1 expression was the highest in 143B cell line, we chose 143B cells for the subsequent experiments. Furthermore, we used the Cancer Cell Line Encyclopedia (CCLE) and found that NUSAP1 was highly expressed in osteosarcoma cell lines (Figure 1C). These data suggested that NUSAP1 may be related to the progression of osteosarcoma.

\section{Effects of NUSAPI on Cell Proliferation of Osteosarcoma I43B Cells}

To explore the functions of NUSAP1 in osteosarcoma cells, NUSAP1 overexpressed and silenced cell models were constructed by transfection with Ov-NUSAP1 or shRNA-NUSAP1 into 143B cells. The transfection efficiency was measured by RT-qPCR and Western blot assay (Figure 2A and B). Next, the effects of NUSAP1 on143B cell proliferation were determined with CCK-8 assay and BrdU assay. As shown in Figure 2C, OD value was significantly increased in Ov-NUSAP1 group compared with the negative control and the control group. However, transfection with shRNA-NUSAP1-1 suppressed the ability of cell proliferation when compared with shRNA-NC group and the control group. In addition, according to the EdU incorporation assay, the number of EdU-positive cells in the cells transfected with Ov-NUSAP1 was dramatically increased while the number of EdU-positive cells in cells transfected with shRNA-NUSAP1-1 was remarkably less than that of the cells in negative control group (Figure 2D). The result indicated that the overexpression or knockdown of NUSAP1 affected the proliferation of 143B cells.

\section{Effects of NUSAPI on Tumor Growth of Osteosarcoma in vivo}

To further investigate the influence of NUSAP1 on cell proliferation of osteosarcoma in vivo, an osteosarcoma 
A

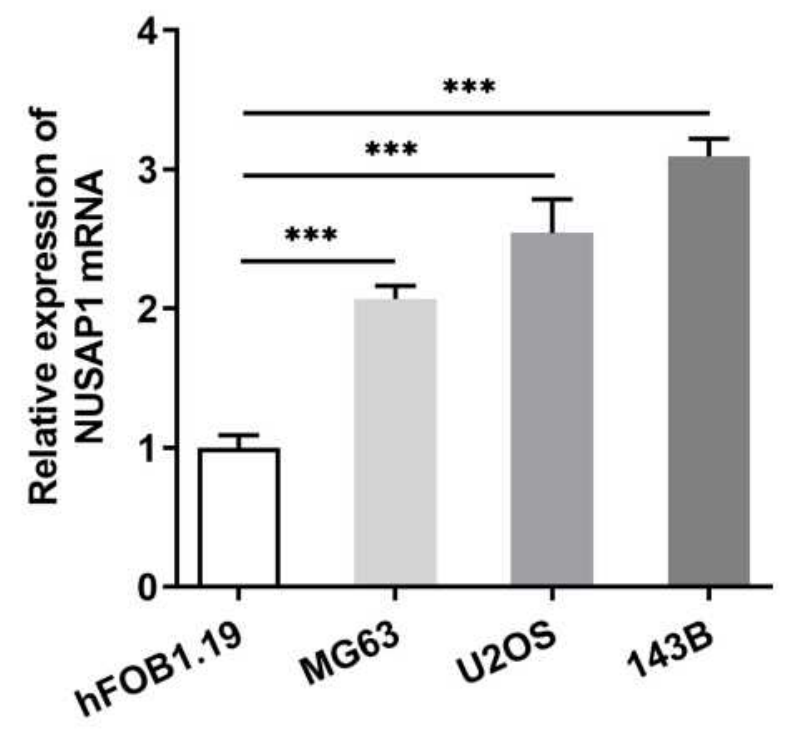

B NUSAP1

GAPDH

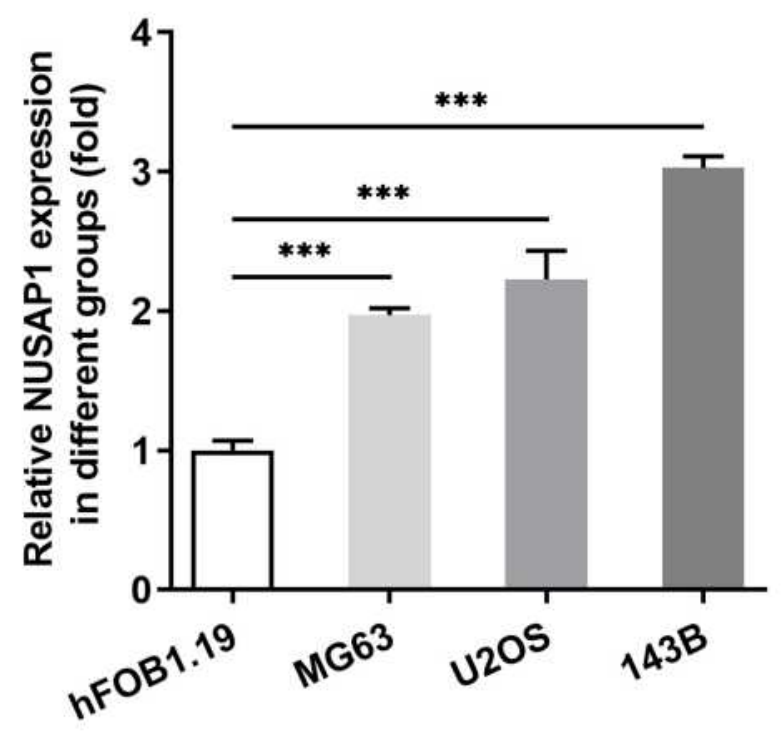

C

mRNA expression (RNAseq): NUSAP1

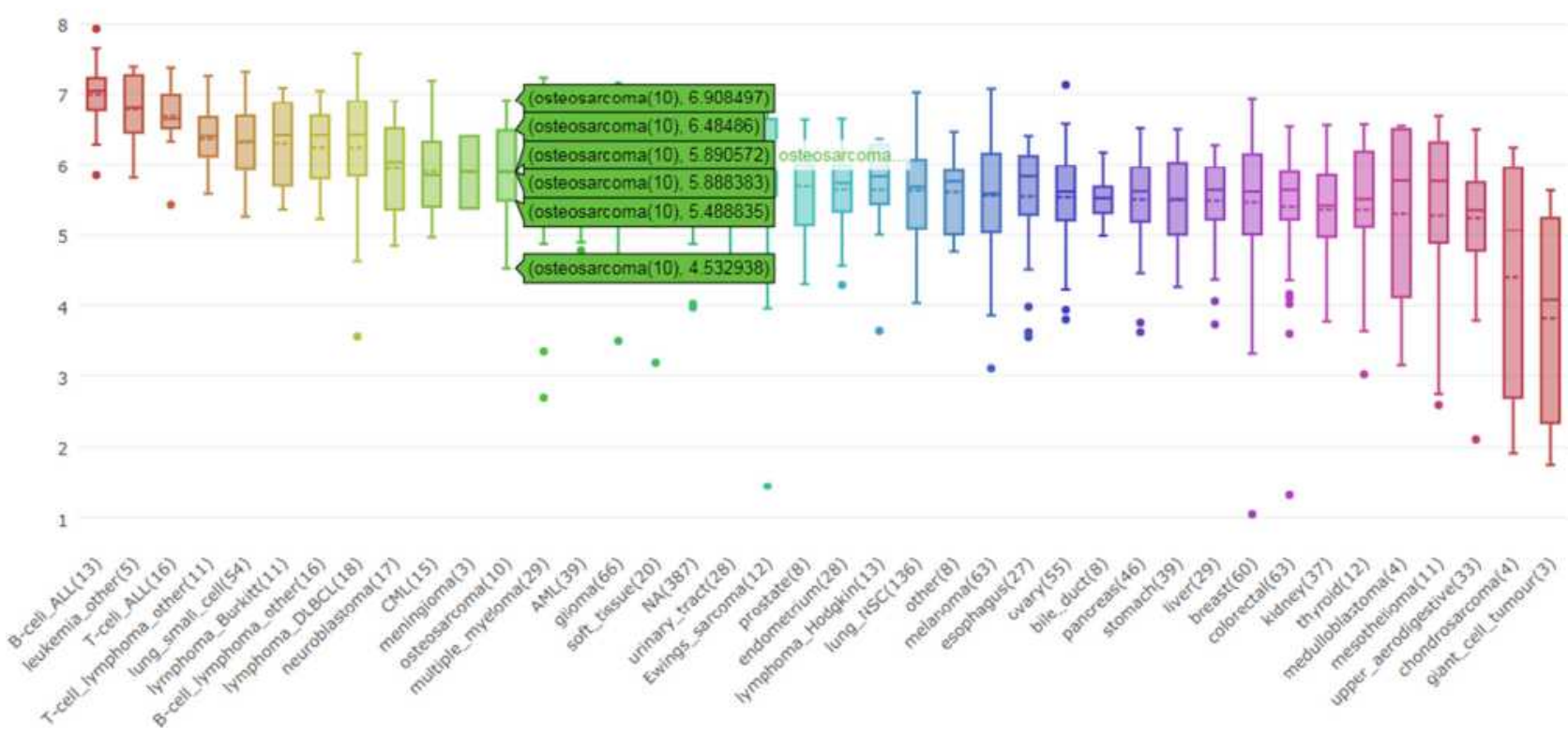

Figure I NUSAPI was highly expressed in osteosarcoma cell lines. (A) RT-qPCR analysis was used to detect NUSAPI mRNA in osteosarcoma cell lines MG63, U2OS and I43B and human normal osteoblast cell line hFOBI.I9. (B) Western blot assay was performed to measure the protein expression of NUSAPI in the three osteosarcoma cell lines and control cells. (C) The expression level of NUSAPI in osteosarcoma cell lines. Data were derived with permission from The Broad Institute of MIT \& Harvard. Cancer Cell Line Encyclopedia (CCLE). Available from: http://www.broadinstitute.org/ccle. ${ }^{33}$ Results are the mean \pm SD. $* * * P<0.00 \mathrm{I}$ versus $\mathrm{hFOB}$ I.I9.

mouse model was established by injection 143B cells transfected with shRNA-NUSAP1-1 or Ov-NUSAP1 (Figure 3A). As shown in Figure $3 \mathrm{~B}$ and $\mathrm{C}$, Nevertheless, shRNA-NUSAP1-1 resulted in decreased overexpression of NUSAP1 significantly increased the tumor volume of mice compared with the control. 

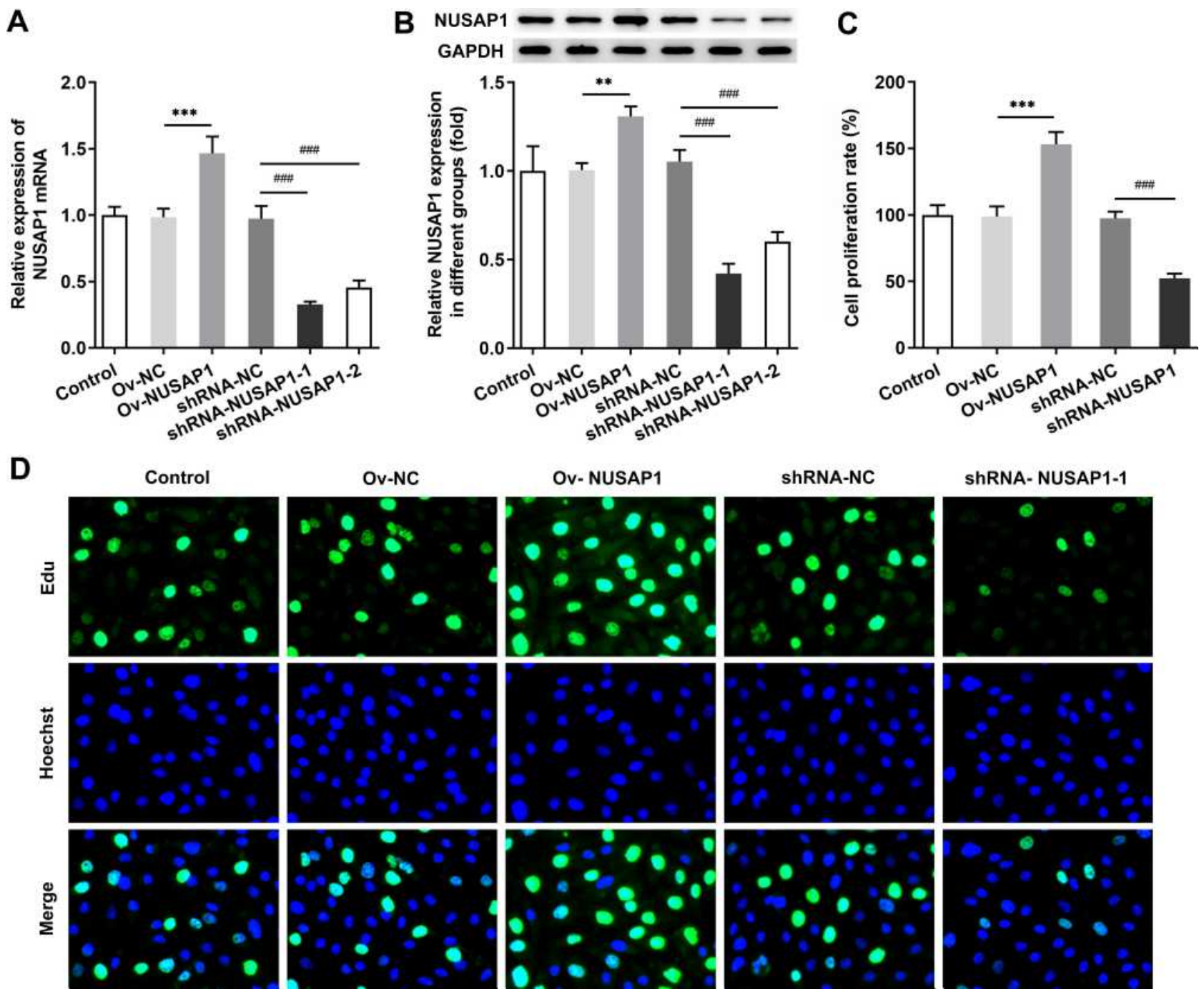

Figure 2 Effects of NUSAPI overexpression or silencing on proliferative ability of I43B cells. RT-qPCR (A) and Western blot assay (B) were utilized to evaluate NUSAPI expression in control or Ov-NUSAPI/shRNA-NUSAPI-I transfected osteosarcoma cells. CCK-8 assay (C) and BrdU assay (D) were performed to identify cell proliferation

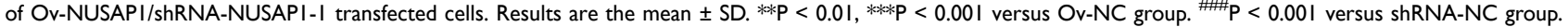

tumor volume in mice. Additionally, Western blot results revealed elevated protein levels of Ki67, PCNA and NUSAP1 in mice transfected overexpressed NUSAP1. However, knockdown of NUSAP1 repressed the productions of the three proteins in mice (Figure 3D). The data indicated the association between NUSAP1 and tumor aggressiveness in osteosarcoma in vivo.

\section{Effects of NUSAPI on Cell Cycle of I43B Cells}

Cell cycling is connected with cancer cell proliferation and G2/M arrest contributes to suppression of proliferation. ${ }^{15}$ To evaluate the impact of NUSAP1 expression on the cell cycling of 143B cells, flow cytometric analysis was performed. As presented in Figure 4, upregulation of NUSAP1 reduced the
G1 phase population and enhanced the $\mathrm{S}$ phase population, compared with those in negative control. However, the percentage of cells in $\mathrm{S}$ phase was decreased and the fractions of cells in G1 phases were increased in shRNA-NUSAP1-1 group. The data suggested that NUSAP1 overexpression promoted cell cycle progression while NUSAP1 silencing inhibited cell proliferation by blocking cell cycle.

\section{NUSAPI Regulates the Expressions of CDC20 and CCNA2}

Next, we implemented the bioinformatics analysis to investigate the molecule mechanisms of NUSAP1-induced effects on osteosarcoma cells. Using STRING database (https:// string-db.org/), we predicted the combination of NUSAP1 with CDC20 and CCNA2 (Figure 5A). Then, RT-qPCR and 
A

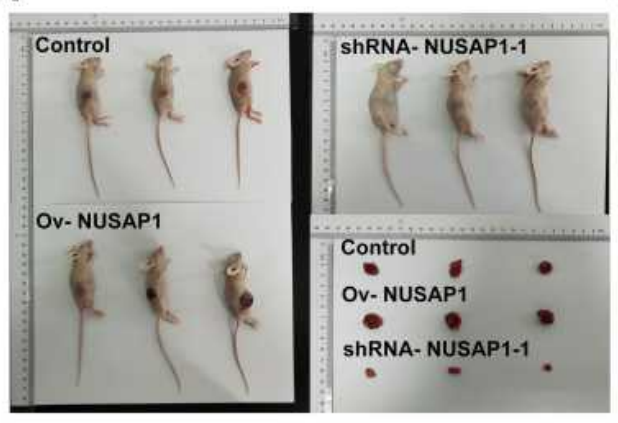

B

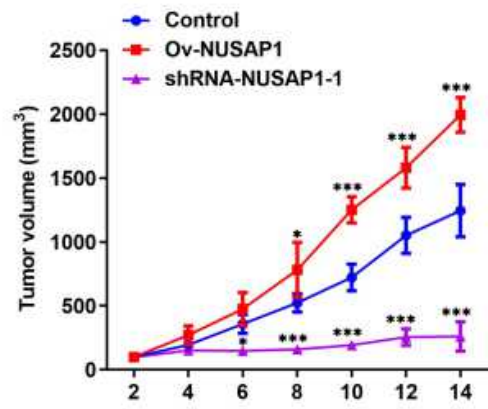

C

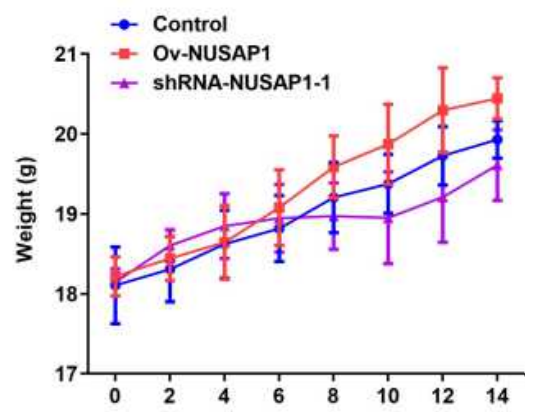

D
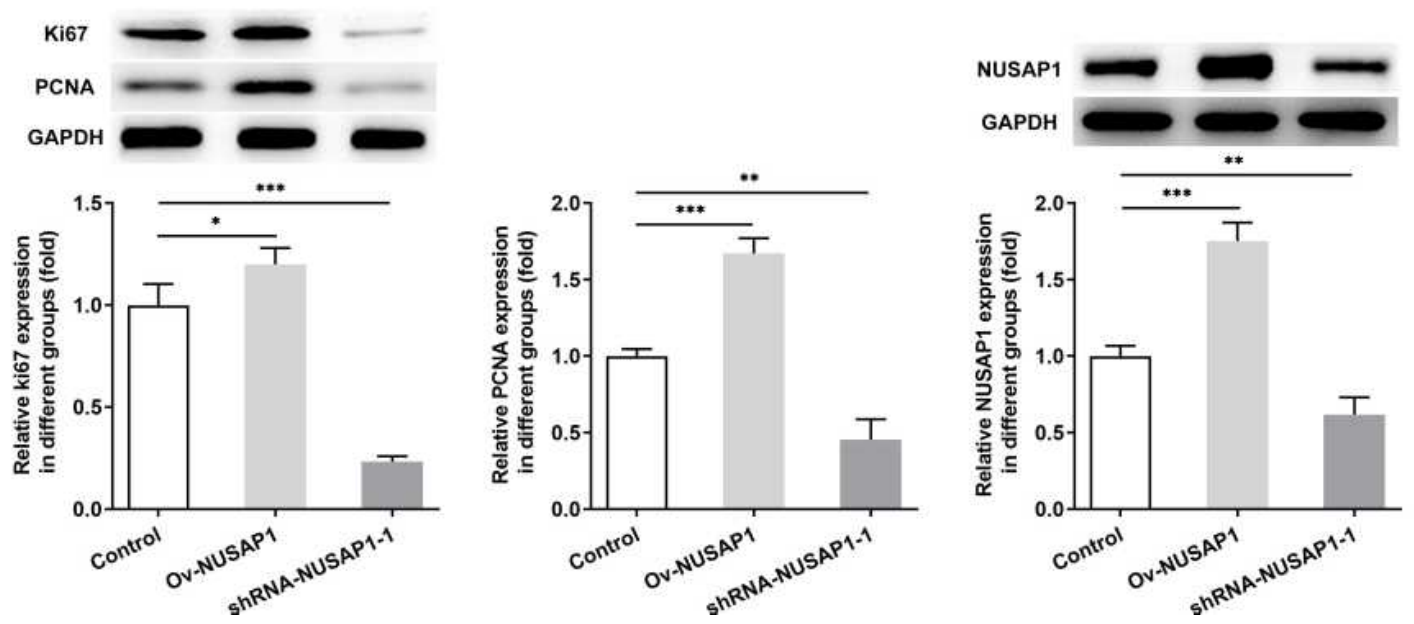

Figure 3 NUSAPI affected osteosarcoma tumor growth in vivo. (A) Xenografted animals were established by injection with osteosarcoma cells transfected with OvNUSAPI/shRNA-NUSAPI-I. Tumor volume (B) and body weight (C) of mouse models were measured every two days for two weeks. (D) Western blotting analysis was carried out to detect the protein expressions of NUSAPI, Ki67 and PCNA. Results are the mean $\pm \mathrm{SD}$. $* \mathrm{P}<0.05, * * \mathrm{P}<0.0 \mathrm{I}, * * * \mathrm{P}<0.001$ versus control.

immunoblotting assay were carried out to verify the prediction. As shown in Figure $5 \mathrm{~B}$ and $\mathrm{C}$, overexpression of NUSAP1 significantly increased the mRNA and protein expressions of CDC20 and CCNA2 but transfection with shRNA-NUSAP1-1 displayed the opposite effects in 143B cells. Also, the immunoprecipitation result showed that CDC20 and CCNA2 were present with the anti-NUSAP1 antibody in the IP assay but not with the control IgG (Figure 5D). The proteins of NUSAP1, CCNA2 and CDC20 were detected with the anti-CDC20 and anti-CCNA2 antibody in the IP assay (Figure 5E and F). The findings suggested that NUSAP1 might combine with CCNA2 and CDC20, and regulated the expressions of them in osteosarcoma.

\section{CDC20 and CCNA2 Silencing Inhibits}

\section{Cell Proliferation and Cell Cycle in} NUSAPI-Mediated I43B Cells

To evaluate the influence of CDC20 and CCNA2 on NUSAP1-mediated osteosarcoma cells, we transfected
shRNA-CDC20-1/2 or shRNA-CCNA2-1/2 into 143B cells and detect the efficiency (Figure 6A and $\mathrm{B}$ ). Then, cell proliferation was assessed by $\mathrm{CCK}-8$ and EdU assay. As illustrated in Figure 6C and D, capacity of cell proliferation was greatly improved in NUSAP1-overexpressed cells while shRNA-CDC20-1 or shRNA-CCNA2-1 reversed the effects of NUSAP1 on proliferation of $143 \mathrm{~B}$ cells. In addition, flow cytometric analysis showed that the $\mathrm{G} 1$ phase population was reduced and the percentage of cells in S and G2/M phase was increased by overexpression of NUSAP1. Nevertheless, transfection with shRNA-CDC20-1 or shRNA-CCNA2-1 elevated the fractions of cells in $\mathrm{G} 1$ phases but reduced the percentage of cells in $\mathrm{S}$ and $\mathrm{G} 2 / \mathrm{M}$ phase in NUSAP1mediated 143B cells (Figure 7). These data revealed the inhibitory effects of CDC2 0 and CCNA2 on cell proliferation and cell cycle in NUSAP1-overexpressed 143B cells.

\section{Discussion}

Osteosarcoma derives from primitive bone-forming mesenchymal cells. It can usually bring about intense 


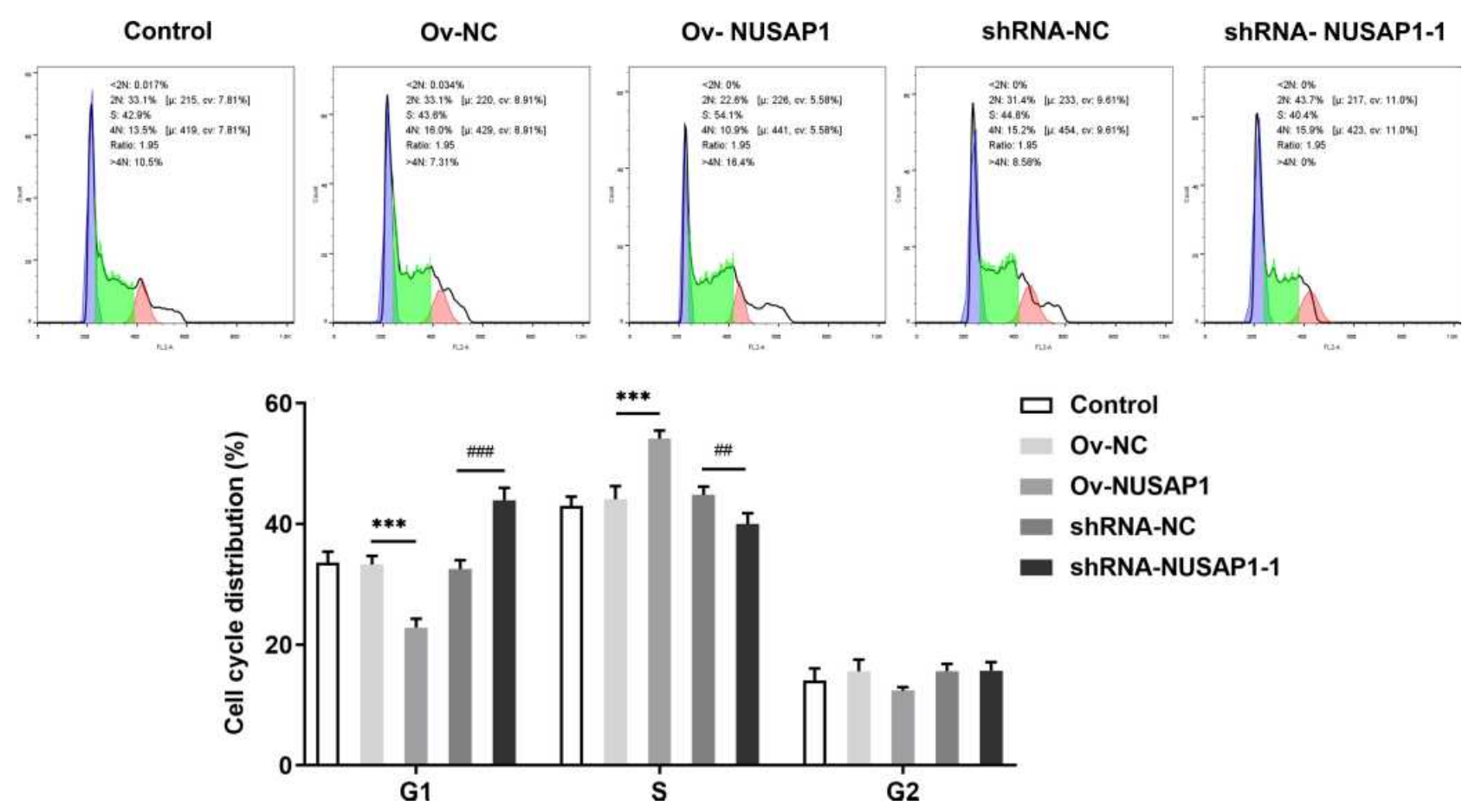

Figure 4 Influence of NUSAPI on cell cycle progression of I43B cells. Flow cytometric analysis was employed to measure the percentage of cells in GI, S and G2/M phase after transfection of Ov-NUSAPI or shRNA-NUSAPI-I. Results are the mean \pm SD. ${ }^{* * *}$ P $<0.00$ I versus Ov-NC group. ${ }^{\# \# P<0.0 I, ~}{ }^{\# \#} \mathrm{P}<0.00 \mathrm{I}$ versus shRNA-NC group.

pain and metastasis to other organs, leading to poor patient prognosis. ${ }^{5,16}$ In this study, we demonstrated that NUSAP1 was highly expressed in several osteosarcoma cell lines. NUSAP1 was associated with osteosarcoma proliferation both in vitro and in vivo. In addition, we also revealed the regulatory effect of NUSAP1 on cell cycling of osteosarcoma and the combination of NUSAP1 with CDC20 and CCNA2. Moreover, inhibition of $\mathrm{CDC} 20$ and CCNA2 reversed the NUSAP1-induced aberrant proliferation and cell cycle progress. These findings provide evidence for carcinogenesis of NUSAP1 in osteosarcoma and revealed the potential mechanism underlying NUSAP1-mediated osteosarcoma.

NUSAP1 plays significant roles in the stability and accuracy of mitotic progression in organisms. ${ }^{17}$ Accumulating evidence have indicated that abnormal expression of NUSAP1 was implicated in tumorigenesis, drug resistance and patient survival. ${ }^{18-20} \mathrm{Li}$ et al reported that NUSAP1 contributed to cervical cancer via stimulating cancer stem cell (CSC) traits and facilitating epithelialmesenchyme transition (EMT) progression via $\mathrm{Wnt} / \beta$ catenin signaling. ${ }^{21}$ Furthermore, Okamoto et al found that NUSAP1 expression was upregulated in Oral Squamous Cell Carcinoma (OSCC)-derived cell lines and knockdown of NUSAP1 inhibited cell proliferation and also promoted anti-tumor effect of Paclitaxel by inducing apoptosis in OSCC. ${ }^{22}$ In addition, the involvement of NUSAP1 in bladder cancer has been demonstrated on account of the effects of NUSAP1 knockdown or overexpression on cell proliferation, migration, invasiveness and chemosensitivity to gemcitabine by regulating the activation of TGF- $\beta$ Signaling Pathway. ${ }^{23}$

In the present study, we examined NUSAP1 expression in some osteosarcoma cell lines and discovered the upregulation of NUSAP1 in these cells compared with normal osteoblast cells, which consistent with previously published findings. However, we did not explore the expression of NUSAP1 gene in bone mesenchymal cells-derived tumor cells and we are exploring the expression and biological effects of NUSAP1 in bone mesenchymal cellsderived tumor cell lines and investigating the potential mechanism in our next study. Moreover, NUSAP1 was revealed to be a facilitator in osteosarcoma proliferation and cell cycle in vitro. We transfected NUSAP1overexpressed plasmid into 143B cells to verify the results above. The data revealed that $143 \mathrm{~B}$ cell proliferation and cell cycle progression were indeed stimulated by upregulation of NUSAP1, while NUSAP1 silencing have inverse effects. Furthermore, to investigate whether NUSAP1 affects osteosarcoma in vivo, we constructed an 
A

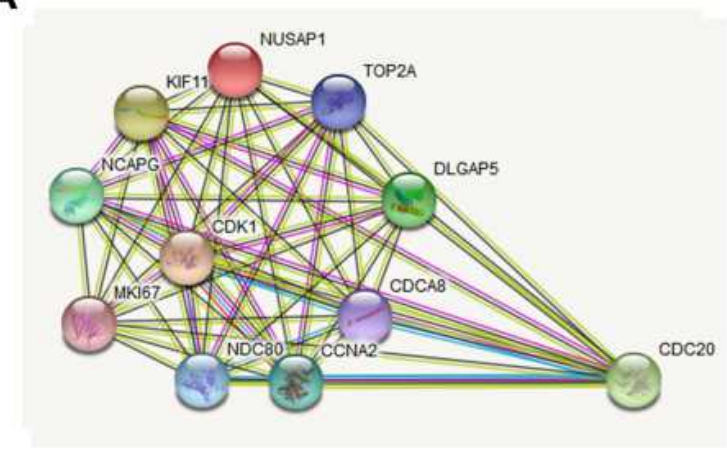

B

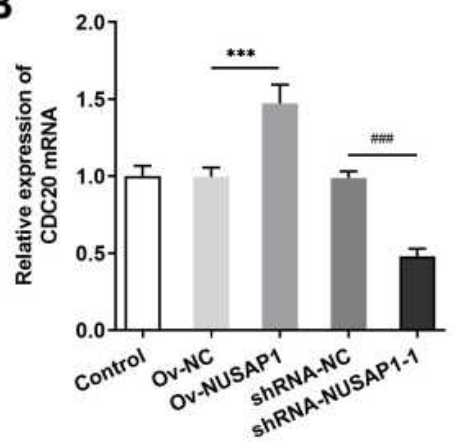

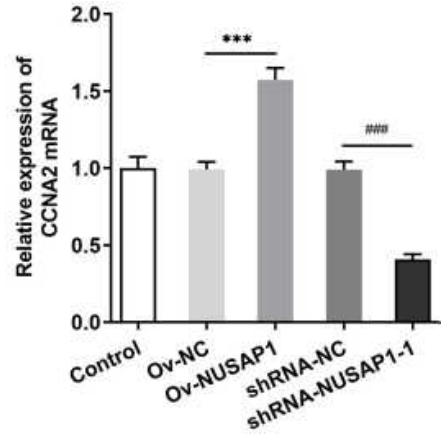

C
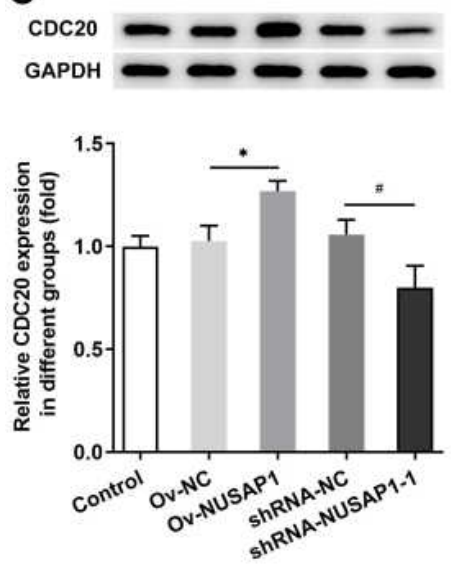
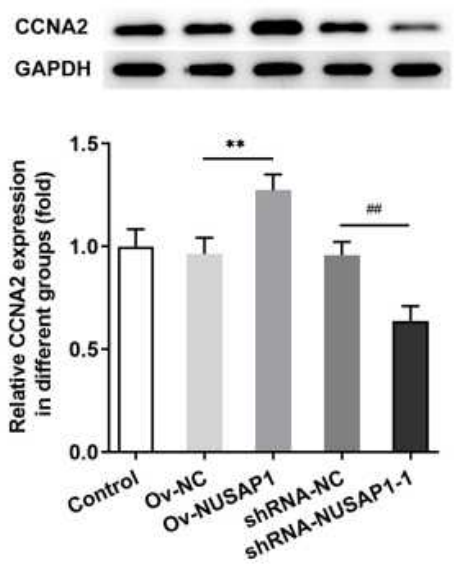

D

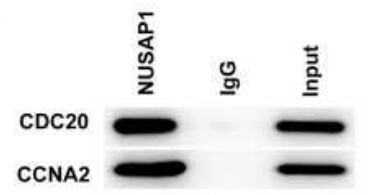

E

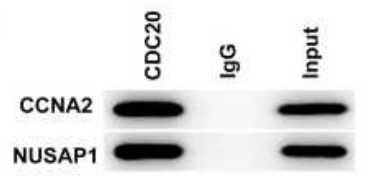

$\mathbf{F}$

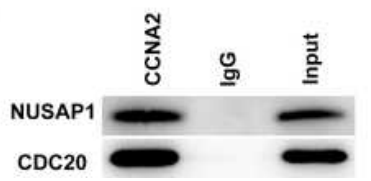

Figure 5 The combination of NUSAPI with CDC20 and CCNA2 in I43B cells. (A) STRING database (https://string-db.org/) was used to predict the relationship between NUSAPI, CDC20 and CCNA2. (B and C) The mRNA and protein expressions of CDC20 and CCNA2 in I43B cells were investigated by RT-qPCR and immunoblotting assay after transfection with control or Ov-NUSAPI/shRNA-NUSAPI-I. (D-F) Immunoprecipitation (IP) assay was performed to verify the combination of NUSAPI with CDC20 and CCNA2. Results are the mean \pm SD. $* \mathrm{P}<0.05, * * \mathrm{P}<0.01$, $* * * \mathrm{P}<0.001$ versus Ov-NC group. ${ }^{\#} \mathrm{P}<0.05,{ }^{\#} \mathrm{P}<0.01,{ }^{\# \#} \mathrm{P}<0.001$ versus shRNA-NC group.

osteosarcoma mouse model and observed that the volume of tumors was intervened after treated with NUSAP1overexpressed or NUSAP1-silenced cell group compared with the untreated tumor-bearing mice. Therefore, the results of the study indicated the oncogenic impact of NUSAP1 on development of osteosarcoma. Thus, NUSAP1 could be used to predict postoperative recurrentmetastasis and serve as a crucial therapeutic target for osteosarcoma.

It has received increasing attention in cell cyclerelated proteins for the role of these dysregulated proteins in many types of diseases, especially in cancers. ${ }^{24-26}$ CDC20 is a vital conserved cell cycle regulator that modulates ubiquitin ligase activity of the APC/C complex at the moment of the metaphase-anaphase transition in mitosis and degrades the substrates of $\mathrm{APC} / \mathrm{C}^{\mathrm{CDC} 20}$ including securin and cyclin A. ${ }^{27,28}$ Previous researches have revealed that $\mathrm{CDC} 20$ suppressed apoptosis while accelerated the proliferation and invasion of osteosarcoma cells. ${ }^{29,30}$ Cyclin A2, as part of the substrates of $\mathrm{APC} / \mathrm{C}^{\mathrm{CDC} 20}$, is tightly linked with the process of sister chromatid segregation. Wu et al analyzed the microarray data GSE42352 and found that two mitosis metaphase to anaphase-related genes CDC20 and cyclin A2 were expressed highly in osteosarcoma cells. ${ }^{31}$ Additionally, Shekhar reported that miR-449a and miR424 were downregulated but cyclin A2 was notably upregulated in clinic osteosarcoma samples. The two microRNAs inhibited aggressive osteosarcoma by cyclin A2 repression. ${ }^{32}$ Our study predicted a potential combination between NUSAP1, CDC20 and cyclin A2 by bioinformatics analysis. According to the results of RTqPCR and Western blot, the expressions of CDC20 and cyclin A2 were changed by knockdown or upregulation NUSAP1 in osteosarcoma cells. In addition, immunoprecipitation assay confirmed the relationship between NUSAP1, CDC20 and cyclin A2. We further investigated the mechanism by which NUSAP1 plays a role in 
A
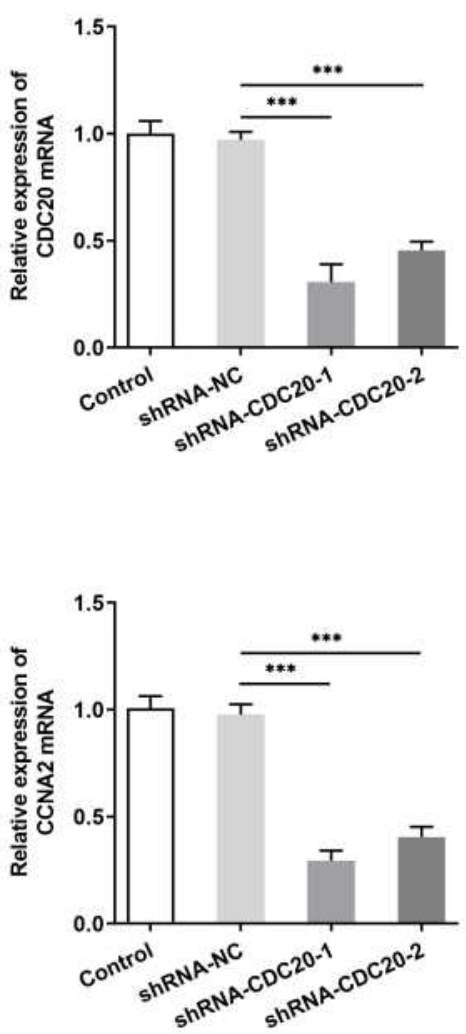

B $\operatorname{CDC}_{20}$

GAPDH

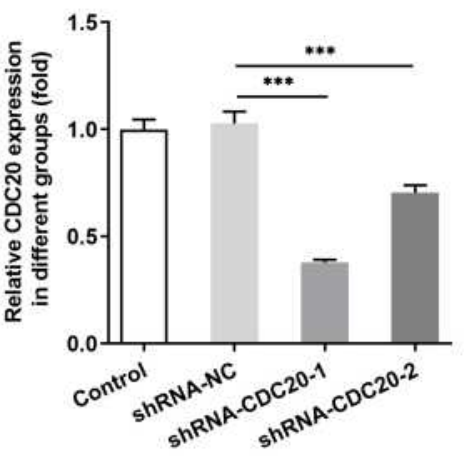

CCNA2 GAPDH

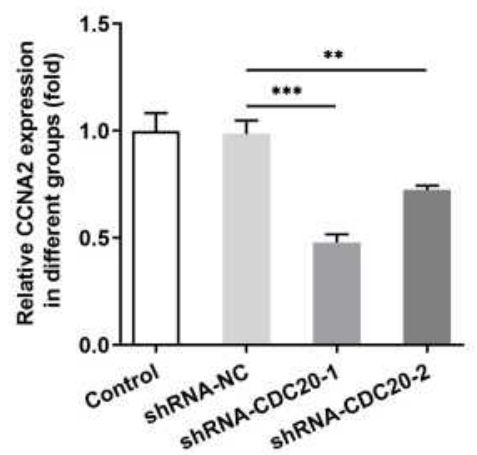

C

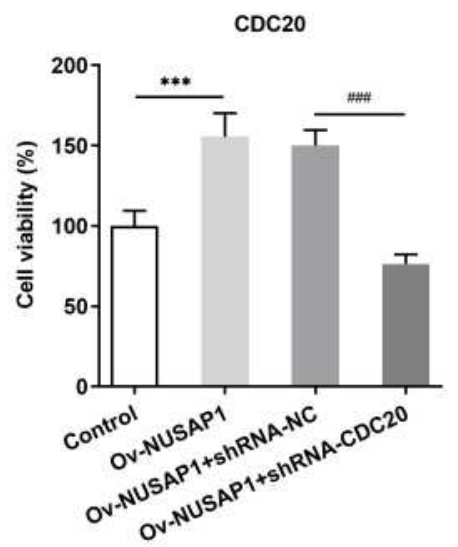

CCNA2

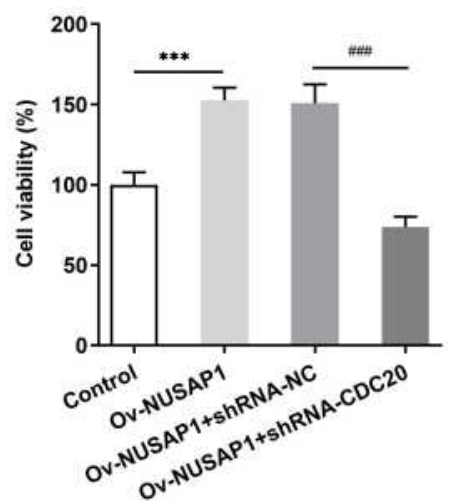

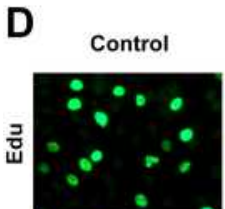

Ov-NUSAP1

Ov-NUSAP1
+shRNA-NC

Ov-NUSAP1
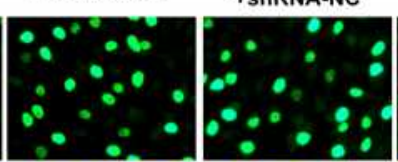

+shRNA-CDC20
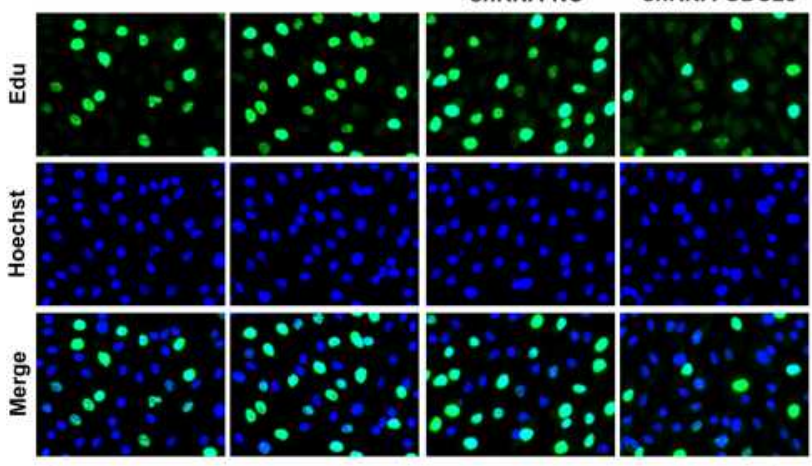

$\operatorname{coc} 20$

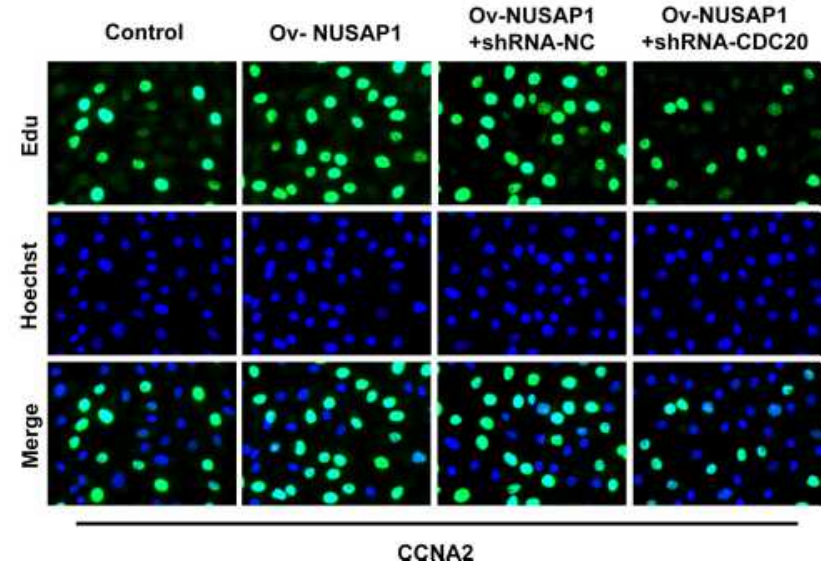

Figure 6 CDC20 and CCNA2 silencing rescued NUSAPI-mediated cell proliferation and cell cycle in I43B cells. (A and B) RT-qPCR assay was implemented to measure transfection efficiency in cells transfected with shRNA-CDC20-I/2 or shRNA-CCNA2-I/2. Results are the mean \pm SD. ***P $<0.01$, ****P $<0.00$ I versus shRNA-NC group. CCK-8 assay (C) and BrdU assay (D) were applied for evaluating cell proliferation ability of shRNA-CDC20-Ior shRNA-CCNA2-I transfected cells. Results are the mean \pm SD. ${ }^{* * * P}<0.001$ versus control. ${ }^{\# \#} \mathrm{P}<0.001$ versus Ov-NUSAPI+shRNA-NC group.

osteosarcoma by binding to CDC20 and cyclin A2. The results revealed downregulation of $\mathrm{CDC} 20$ and cyclin $\mathrm{A} 2$ rescued the NUSAP1-mediated malignant properties of osteosarcoma cells by cell proliferation inhibition and cell cycle arrest.

\section{Conclusions}

Taken together, NUSAP1 exerts oncogenic roles in osteosarcoma with accelerated cell proliferation and relieving the blockage of cell cycle. Importantly, silencing of CDC20 and cyclin A2 that were regulated by NUSAP1 

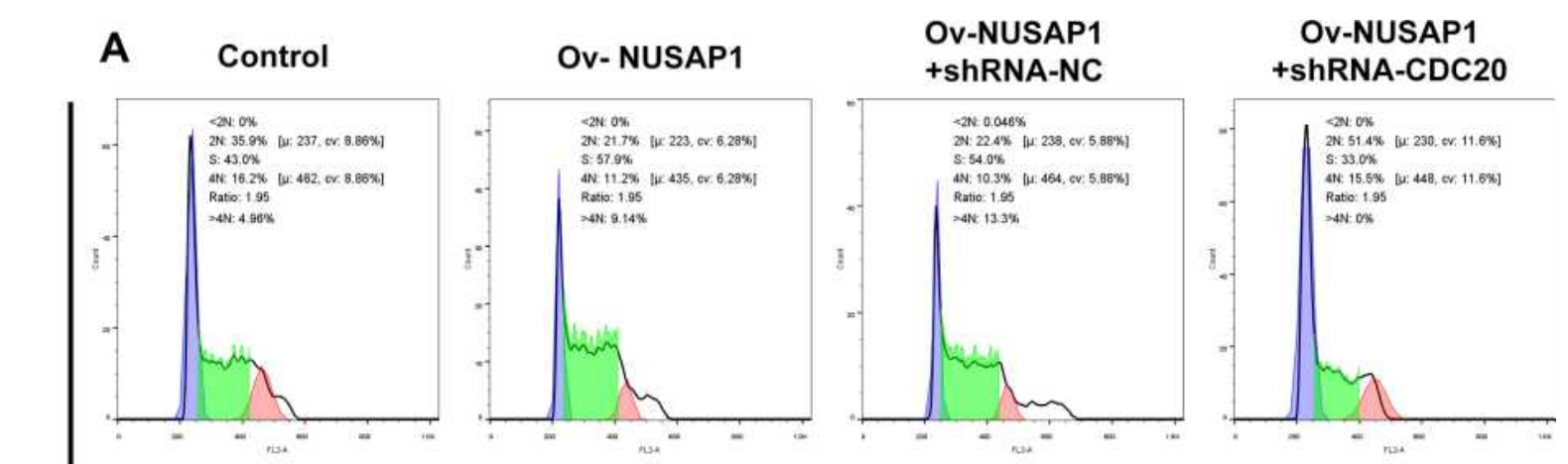

ญัญ
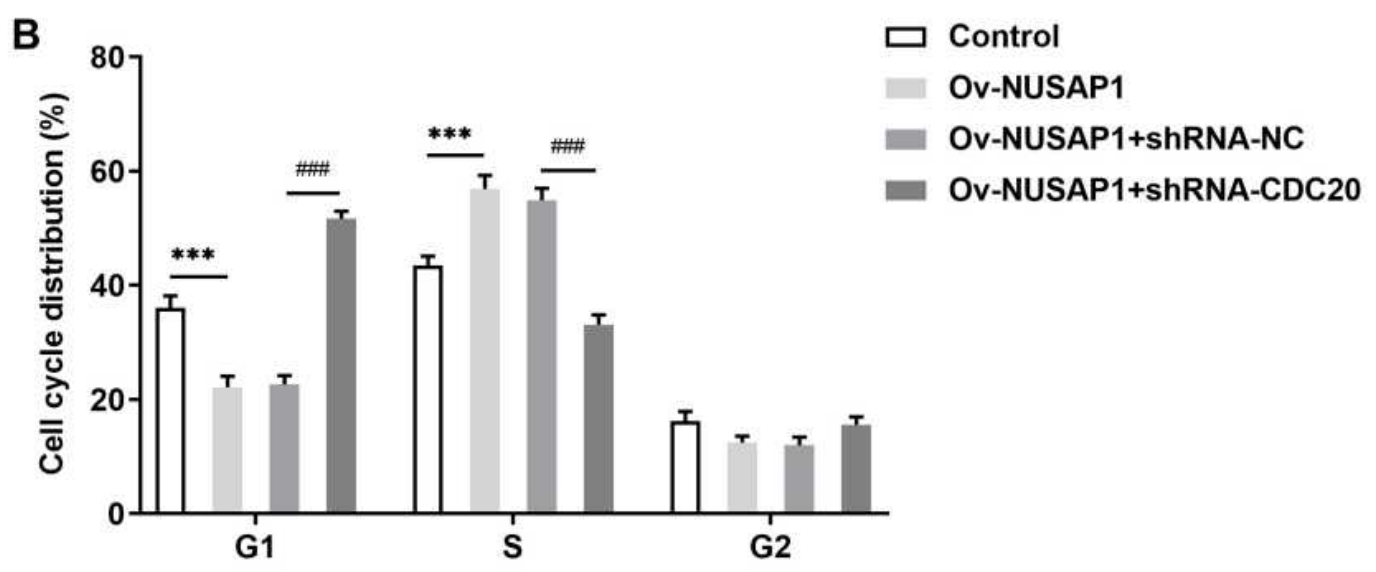

C Control

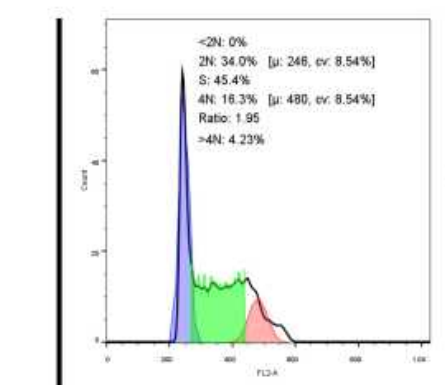

\section{Ov- NUSAP1}

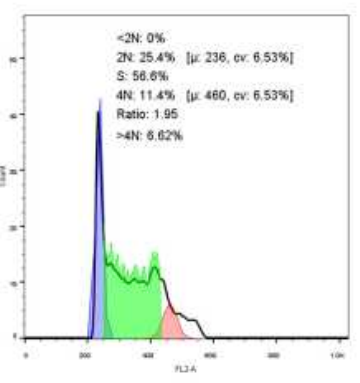

\্য

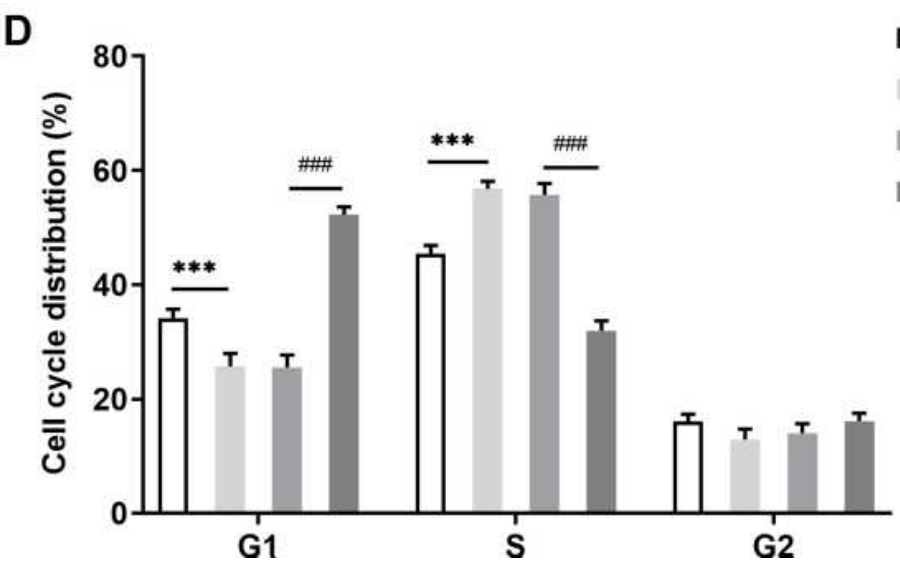

Figure 7 Influence of CDC20 and CCNA2 on cell cycle progression of I43B cells. (A and B) Flow cytometric analysis was employed to detect the fractions of cells in GI, $\mathrm{S}$ and G2/M phase after transfection of shRNA-CDC20. (C and D) Cell cycle of I43B cells transfected with shRNA-CCNA2 was detected by flow cytometric analysis. Results are the mean \pm SD. ${ }^{* * * P}<0.001$ versus control. ${ }^{\# \# P}<0.001$ versus Ov-NUSAPI+shRNA-NC group. 
may serve as an alternative special therapy for osteosarcoma patients.

\section{Disclosure}

The authors report no conflicts of interest in this work.

\section{References}

1. Harrison DJ, Geller DS, Gill JD, et al. Current and future therapeutic approaches for osteosarcoma. Expert Rev Anticancer Ther. 2018;18 (1):39-50. doi:10.1080/14737140.2018.1413939

2. Müller DA, Silvan U. On the biomechanical properties of osteosarcoma cells and their environment. Int J Dev Biol. 2019;63(1-2):1-8. doi:10.1387/ijdb.190019us

3. Anderson ME. Update on survival in osteosarcoma. Orthop Clin North Am. 2016;47(1):283-292. doi:10.1016/j.ocl.2015.08.022

4. Isakoff MS, Bielack SS, Meltzer P, et al. Osteosarcoma: current treatment and a collaborative pathway to success. J Clin Oncol. 2015;33(27):3029-3035. doi:10.1200/JCO.2014.59.4895

5. Simpson E, Brown HL. Understanding osteosarcomas. JAAPA. 2018;31(8):15-19. doi:10.1097/01.JAA.0000541477.24116.8d

6. Bishop MW, Janeway KA, Gorlick R. Future directions in the treatment of osteosarcoma. Curr Opin Pediatr. 2016;28(1):26-33. doi:10.1097/MOP.0000000000000298

7. Mills CA, Suzuki A, Arceci A, et al. Nucleolar and spindle-associated protein 1 (NUSAP1) interacts with a SUMO E3 ligase complex during chromosome segregation. $J$ Biol Chem. 2017;292(42):17178-17189. doi:10.1074/jbc.M117.796045

8. Han G, Wei Z, Cui H, et al. NUSAP1 gene silencing inhibits cell proliferation, migration and invasion through inhibiting DNMT1 gene expression in human colorectal cancer. Exp Cell Res. 2018;367(2):216-221. doi:10.1016/j.yexcr.2018.03.039

9. Xu Z, Wang Y, Xiong J, et al. NUSAP1 knockdown inhibits cell growth and metastasis of non-small-cell lung cancer through regulating BTG2/PI3K/Akt signaling. $J$ Cell Physiol. 2020;235 (4):3886-3893. doi:10.1002/jcp.29282

10. Gordon CA, Gong X, Ganesh D, et al. NUSAP1 promotes invasion and metastasis of prostate cancer. Oncotarget. 2017;8 (18):29935-29950. doi:10.18632/oncotarget.15604

11. Yang Z, Jiong L, Guoxing F, et al. Hepatitis B virus X protein enhances hepatocarcinogenesis by depressing the targeting of NUSAP1 mRNA by miR-18b. Cancer Biol Med. 2019;16 (2):276-287. doi:10.20892/j.issn.2095-3941.2018.0283

12. Liu X, Chen Y, Li Y, et al. Targeting mitosis exit: a brake for cancer cell proliferation. Biochim Biophys Acta Rev Cancer. 2019;1871 (1):179-191. doi:10.1016/j.bbcan.2018.12.007

13. Chen L, Yang L, Qiao F, et al. High levels of nucleolar spindle-associated protein and reduced levels of BRCA1 expression predict poor prognosis in triple-negative breast cancer. PLoS One. 2015;10(10):e0140572. doi:10.1371/journal.pone.0140572

14. Roy S, Hooiveld GJ, Seehawer M, et al. microRNA 193a-5p regulates levels of nucleolar- and spindle-associated protein 1 to suppress hepatocarcinogenesis. Gastroenterology. 2018;155(6):1951-1966. e26. doi:10.1053/j.gastro.2018.08.032

15. Icard P, Fournel L, Wu Z, et al. Interconnection between metabolism and cell cycle in cancer. Trends Biochem Sci. 2019;44(6):490-501. doi:10.1016/j.tibs.2018.12.007

16. Xie L, Ji T, Guo W. Anti-angiogenesis target therapy for advanced osteosarcoma (review). Oncol Rep. 2017;38(2):625-636. doi:10.3892/or.2017.5735
17. Kotian S, Banerjee T, Lockhart A, et al. NUSAP1 influences the DNA damage response by controlling BRCA1 protein levels. Cancer Biol Ther. 2014;15(5):533-543. doi:10.4161/cbt.28019

18. Zhang X, Pan Y, Fu H, Zhang J. Nucleolar and spindle associated protein 1 (NUSAP1) inhibits cell proliferation and enhances susceptibility to epirubicin in invasive breast cancer cells by regulating cyclin D kinase (CDK1) and DLGAP5 expression. Med Sci Monit. 2018;24:8553-8564. doi:10.12659/MSM.910364

19. Zhao Y, He J, Li Y, et al. NUSAP1 potentiates chemoresistance in glioblastoma through its SAP domain to stabilize ATR. Signal Transduct Target Ther. 2020;5(1):44. doi:10.1038/s41392-020-0137-7

20. Xie Q, Ou-yang W, Zhang M, et al. Decreased expression of NUSAP1 predicts poor overall survival in cervical cancer. $J$ Cancer. 2020;11(10):2852-2863. doi:10.7150/jca.34640

21. Li H, Zhang W, Yan M, et al. Nucleolar and spindle associated protein 1 promotes metastasis of cervical carcinoma cells by activating Wnt/ß-catenin signaling. J Exp Clin Cancer Res. 2019;38(1):33. doi:10.1186/s13046-019-1037-y

22. Okamoto A, Higo M, Shiiba M, et al. Down-regulation of nucleolar and spindle-associated protein 1 (NUSAP1) expression suppresses tumor and cell proliferation and enhances anti-tumor effect of paclitaxel in oral squamous cell carcinoma. PLoS One. 2015;10(11): e0142252. doi:10.1371/journal.pone.0142252

23. Gao S, Yin H, Tong H. Nucleolar and spindle associated protein 1 (NUSAP1) promotes bladder cancer progression through the TGF- $\beta$ signaling pathway. Onco Targets Ther. 2020;13:813-825. doi:10.2147/OTT.S237127

24. Shostak A. Circadian clock, cell division, and cancer: from molecules to organism. Int $J$ Mol Sci. 2017;18(4):873. doi:10.3390/ ijms 18040873

25. Zheng K, He Z, Kitazato K, et al. Selective autophagy regulates cell cycle in cancer therapy. Theranostics. 2019;9(1):104-125. doi: $10.7150 /$ thno. 30308

26. Chang WH, Forde D, Lai AG. Dual prognostic role of 2-oxoglutarate-dependent oxygenases in ten cancer types: implications for cell cycle regulation and cell adhesion maintenance. Cancer Commun. 2019;39(1):23. doi:10.1186/s40880-019-0369-5

27. Kapanidou M, Curtis NL, Bolanos-Garcia VM. Cdc20: at the crossroads between chromosome segregation and mitotic exit. Trends Biochem Sci. 2017;42(3):193-205. doi:10.1016/j.tibs.2016.12.001

28. Richeson KV, Bodrug T, Sackton KL, et al. Paradoxical mitotic exit induced by a small molecule inhibitor of $\mathrm{APC} / \mathrm{C}(\mathrm{Cdc} 20)$. Nat Chem Biol. 2020;16(5):546-555. doi:10.1038/s41589-020-0495-z

29. Shang G, Ma X, Lv G. Cell division cycle 20 promotes cell proliferation and invasion and inhibits apoptosis in osteosarcoma cells. Cell Cycle. 2018;17(1):43-52. doi:10.1080/15384101.2017.1387700

30. Gao Y, Zhang B, Wang Y, et al. Cdc20 inhibitor apcin inhibits the growth and invasion of osteosarcoma cells. Oncol Rep. 2018;40 (2):841-848. doi:10.3892/or.2018.6467

31. Wu MS, Ma Q-Y, Liu-D-D, et al. CDC20 and its downstream genes: potential prognosis factors of osteosarcoma. Int $J$ Clin Oncol. 2019;24(11):1479-1489. doi:10.1007/s10147-019-01500-3

32. Shekhar R, Priyanka P, Kumar P, et al. The microRNAs miR-449a and miR-424 suppress osteosarcoma by targeting cyclin A2 expression. J Biol Chem. 2019;294(12):4381-4400. doi:10.1074/jbc. RA118.005778

33. The Broad Institute of MIT \& Harvard. Cancer Cell Line Encyclopedia (CCLE). Available from: http://www.broadinstitute. org/ccle. Accessed December 04, 2020. 


\section{Publish your work in this journal}

OncoTargets and Therapy is an international, peer-reviewed, open access journal focusing on the pathological basis of all cancers, potential targets for therapy and treatment protocols employed to improve the management of cancer patients. The journal also focuses on the impact of management programs and new therapeutic agents and protocols on patient perspectives such as quality of life, adherence and satisfaction. The manuscript management system is completely online and includes a very quick and fair peer-review system, which is all easy to use. Visit http://www.dovepress.com testimonials.php to read real quotes from published authors. 